\title{
Poor Decision Making Is Associated with an Increased Risk of Mortality among Community-Dwelling Older Persons without Dementia
}

\author{
Patricia A. Boyle ${ }^{a, b}$ Robert S. Wilson ${ }^{a-c}$ Lei Yu ${ }^{a, c}$ Aron S. Buchman ${ }^{a, c}$ \\ David A. Bennett ${ }^{\mathrm{a}, \mathrm{c}}$ \\ ${ }^{a}$ Rush Alzheimer's Disease Center, ${ }^{b}$ Department of Behavioral Sciences and ${ }^{\mathrm{C}}$ Department of Neurological Sciences, \\ Rush University Medical Center, Chicago, III., USA
}

\begin{abstract}
Background: Decision making is thought to be an important determinant of health and well-being across the lifespan, but little is known about the association of decision making with mortality. Methods: Participants were 675 older persons without dementia from the Rush Memory and Aging Project, a longitudinal cohort study of aging. Baseline assessments of decision making were used to predict the risk of mortality during up to 4 years of follow-up. Results: The mean score on the decision making measure at baseline was 7.1 ( $S D=2.9$, range: $0-12$ ), with lower scores indicating poorer decision making. During up to 4 years of follow-up ( mean $=1.7$ years), 40 (6\% of 675 ) persons died. In a proportional hazards model adjusted for age, sex and education, the risk of mortality increased by about $20 \%$ for each additional decision making error $(\mathrm{HR}=1.19,95 \% \mathrm{Cl}=1.07-1.32$, $p=0.002$ ). Thus, a person who performed poorly on the measure of decision making (score $=3$, 10th percentile) was about 4 times more likely to die compared to a person who performed well (score $=11$, 90th percentile). Further, the association of decision making with mortality persisted after adjustment for the level of cognitive function. Conclusion: Poor decision making is associated with an increased risk of mortality in old age even after accounting for cognitive function.

Copyright $\odot 2013$ S. Karger AG, Basel
\end{abstract}

\section{Introduction}

Decision making involves the ability to understand, generate and evaluate competing alternatives and select an optimal choice. Virtually all behaviors result from some decision making process, from the mundane (e.g. take the stairs versus the elevator) to the complex (e.g. stock portfolio choice), and efficient decision making is thought to be critical for maintaining independence, health and well-being across the lifespan [1-3]. Decision making is particularly salient in aging, the time when many complex and consequential decisions are made (e.g. intergenerational transfers of wealth, end of life healthcare decisions) [4]. It is widely recognized that older persons with overt cognitive impairment (i.e. dementia) exhibit impaired decision making $[5,6]$, and a few recent studies suggest that persons in the preclinical phase of dementia also have difficulty making even routine decisions [7-10]. However, while it is clear that cognition is critical for decision making, decision making also involves factors other than cognitive ability [1-4]. In particular, decision making requires domain-specific knowledge (e.g. investment portfolio decisions require a very different fund of knowledge than that needed to decide whether to pursue a painful and costly treatment for an

\section{KARGER}

E-Mail karger@karger.com

www.karger.com/ned
C) 2013 S. Karger AG, Basel

0251-5350/13/0404-0247\$38.00/0
Patricia Boyle, $\mathrm{PhD}$

Rush Alzheimer's Disease Center

600 S. Paulina, Suite 1020B

Chicago, IL 60612 (USA)

E-Mail Patricia_Boyle@rush.edu 
aggressive cancer unlikely to be cured). In addition, decision making requires personality traits such as patience and self-control; there are many knowledgeable adults with high cognitive capacity that smoke, drink and drive, or have diabetes that could be controlled by diet. Thus, decision making is a complex function of diverse characteristics and may be a harbinger of adverse health outcomes even among persons without overt cognitive impairment. To date, however, relatively few studies have examined decision making in older persons and the consequences of impaired decision making in old age remain unknown.

In this study, we tested the hypothesis that poor decision making is associated with an increased risk of mortality among community-based older persons without dementia. Participants were 675 older persons from the Rush Memory and Aging Project, a longitudinal study of aging. Decision making was assessed using a previously established, 12-item, performance-based measure of decision making that employs materials designed to simulate those used in real world settings and has been related to cognition and health in prior cross-sectional studies of aging $[2,3,11]$. Proportional hazard models adjusted for age, sex and education were used to examine the association of decision making with risk of mortality. In subsequent analyses, to examine the robustness of the relation between decision making and mortality, we added a term to control for the influence of the level of cognitive function based on 19 cognitive tests. This was done because dementia and level of cognition are well known to be associated with risk of death. By excluding persons with dementia at baseline and controlling for level of cognition, we explicitly sought to determine whether factors related to decision making other than cognition are associated with risk of death. Such a finding would have important implications for developing strategies to improve decision making in older persons.

\section{Method}

\section{Participants}

Participants were from the Memory and Aging Project, an ongoing longitudinal study of chronic conditions of aging that began in 1997; enrollment is ongoing [12]. Participation involves risk factor assessment, detailed annual clinical evaluations including medical history, neurological and neuropsychological examinations, and organ donation at death. The study was approved by the Institutional Review Board of Rush University Medical Center, and informed consent and an anatomical gift act were obtained following a detailed presentation of the risks and benefits associated with participation. Notably, in 2008, decision making assessment was started as part of a substudy that was added and was also approved by the Institutional Review Board of Rush.

At the time of these analyses, 1,514 participants had completed the baseline evaluation for the parent study; of those, 418 died and 82 refused further participation in the parent project before they were approached for a decision making assessment. Of the remaining 1,014 potentially eligible persons, 695 completed the decision making assessment, 318 had not yet completed their decision making baseline evaluation and 1 refused the decision making assessment. Of the 695 participants who had completed the decision making assessment, 20 had dementia and were excluded, leaving 675 eligible for these analyses.

\section{Clinical Diagnoses and Cognitive Evaluation}

Clinical diagnoses were performed using a uniform process, as previously described [12]. First, neuropsychological tests were administered and ratings of impairment were assigned based on education-adjusted cutoff scores. Second, an experienced neuropsychologist, blinded to subject age, sex and race, reviewed the results of the cognitive testing and rendered a clinical judgment regarding the presence of cognitive impairment. Third, diagnostic classification was performed by an experienced clinician who specified whether the participant met clinical criteria for dementia and probable Alzheimer's disease recommended by the joint working group of the National Institute of Neurologic and Communicative Disorders and Stroke and the Alzheimer's Disease and Related Disorders Association; these criteria require evidence of cognitive decline in memory and at least one other domain of cognitive function.

Cognitive function was assessed via a battery of 21 tests, including the Mini Mental State Examination, but the latter scores were used only to describe the cohort. One additional test, Complex Ideational Material, is used for diagnostic classification purposes only. Scores on 19 tests were used to create a summary measure of global cognitive function, as previously described.

To compute the composite measure of global cognitive function, raw scores on each of the individual tests were converted to $\mathrm{z}$-scores using the baseline mean and standard deviation of the entire cohort, and the $\mathrm{z}$-scores of all 19 tests were averaged [12-14].

\section{Assessment of Decision Making}

A 12-item version of a previously established performancebased measure was used to examine decision making [2, 3, 11-14]. This measure was specifically designed to evaluate decision making in older adults and uses theoretically based and conceptually well-considered items to assess aspects of decision making such as comprehension and integration of information using materials that closely resemble those used in real world decision settings commonly faced by older persons [2,3]. Respondents are asked questions of varying difficulty levels (simple and complex). Simple questions primarily measure decisions that reflect understanding of the information presented. The complex problems parallel the simple problems but involve many more options. For example, one of the simple problems presents information on three mutual funds, including the gross annual return, account management fee, minimum investment, and years of activity, and asks respondents to select the fund with the smallest account management fee. Subsequently, a complex problem presents sim- 
ilar information about seven mutual funds and asks respondents to select the most appropriate fund given prespecified preferences (e.g. Pamela wants a management fee of less than $\mathrm{X} \%$, a gross annual rate of return of $\mathrm{X} \%$, and a minimum investment of $\mathrm{X}$; which fund should she choose?). The total score is the sum of the number of items answered correctly (range: 0-12), with higher scores indicating better decision making. In previous research, this measure has been shown to have adequate psychometric properties including internal consistency, interrater reliability and shortterm temporal stability. Further, an expanded version of the test has been shown to be related to behaviors and abilities known to be related to decision making in old age, including measures of cognitive ability, numeracy, attitudinal and psychosocial measures (e.g. motivation) and health behaviors (e.g. number of medications taken, number of doctor/nurse visits) $[2,3]$. Finally, in our cohort, performance on the test is related to real world decisions such as susceptibility to scams $(\mathrm{p}<0.001)$ and likelihood of having been a victim of financial fraud $(\mathrm{p}<0.01)$, in addition to other indices that should be related to decision making in old age (e.g. age, education, income, conscientiousness and functional status, all $\mathrm{p}$ values $<0.001)$.

\section{Other Covariates}

Other variables used in the analyses included age (based on date of birth and date of cognitive testing), sex (females coded as 0 and males as 1) and education (years of schooling completed).

\section{Determination of Vital Status}

The autopsy rate of the Rush Memory and Aging Project exceeds $80 \%$. Thus, for most participants, the exact date of death is known. Study participants are also contacted quarterly to determine vital status and changes in health, and death is occasionally learned of during quarterly contacts. Finally, research assistants regularly search the Social Security Death Index via the internet for the small number of persons lost to follow-up. At the time of these analyses, mortality data were accurate within 3 months.

\section{Data Analysis}

We first examined the crude associations of decision making with age, sex and education. Then, we examined the relation of decision making with mortality using a proportional hazards model adjusted for age, sex and education. Finally, to examine the potential influence of cognition on the association of decision making with mortality, we repeated the core model with an additional term for cognitive function. Model validation was performed graphically and analytically and there was no evidence of nonlinearity or nonproportionality. Programming was done in SAS [15].

\section{Results}

\section{Characteristics of Decision Making}

Baseline scores on the decision making measure ranged from 0 to 12 (mean $=7.1, \mathrm{SD}=2.9$ ), with lower scores indicating poorer decision making. Decision mak-
Table 1. Baseline characteristics of participants who survived or died

\begin{tabular}{lccc}
\hline Characteristic & $\begin{array}{l}\text { Survived } \\
(\mathrm{n}=632)\end{array}$ & $\begin{array}{l}\text { Died } \\
(\mathrm{n}=40)\end{array}$ & $\begin{array}{l}\mathrm{p} \\
\text { value }\end{array}$ \\
\hline Age, years & $81.5 \pm 7.3$ & $87.6 \pm 6.3$ & $<0.001$ \\
Sex female & $490(77.2 \%)$ & $26(65 \%)$ & 0.0786 \\
Race white & $588(93.0 \%)$ & $40(100 \%)$ & 0.1005 \\
Education, years & $15.2 \pm 2.9$ & $15.0 \pm 3.1$ & 0.700 \\
Decision making score & $7.3 \pm 2.8$ & $5.0 \pm 3.0$ & $<0.001$ \\
Cognition score & $0.3 \pm 0.5$ & $0.0 \pm 0.5$ & 0.0021
\end{tabular}

Mean values \pm SD are presented unless otherwise noted, and statistical significance is based on t tests (or Mann-Whitney Wilcoxon rank sum) or $\chi^{2}$ tests, as appropriate.

ing was negatively associated with age $(\mathrm{r}=-0.33, \mathrm{p}<$ $0.001)$ and positively with education $(r=0.38, p<0.001)$ such that older and less educated persons exhibited poorer decision making. Women performed more poorly than men $(p=0.004)$. Decision making was also positively associated with cognition $(\mathrm{r}=0.56, \mathrm{p}<0.001)$.

\section{Decision Making and Mortality}

Over up to 4 years of follow-up (mean $=1.7), 40(6 \%$ of $675)$ persons died. Table 1 provides crude data at baseline on those who died and those who survived. Those who died were older and exhibited poorer decision making compared to survivors. In addition, those who died had lower levels of cognitive function.

We examined the association of decision making with the risk of mortality via a series of proportional hazard models adjusted for age, sex and education. In the initial analysis, the risk of mortality increased by about $20 \%$ for each additional error made on the performance-based measure of decision making ( $\mathrm{HR}=1.19,95 \% \mathrm{CI}=1.07$ 1.32). Thus, as illustrated in figure 1 , a person who performed poorly on the decision making measure (score = 3,10 th percentile) was about 4 times more likely to die over the course of the study compared to a person who performed well (score $=11$, 90th percentile).

Next, because it is well known that cognitive function is strongly associated with the risk of mortality in old age and efficient decision making requires cognitive function, we examined whether the association of decision making with mortality persisted after adjustment for cognitive function. Thus, we repeated the initial proportional hazard model described above but included an additional term for global cognitive function, which was 


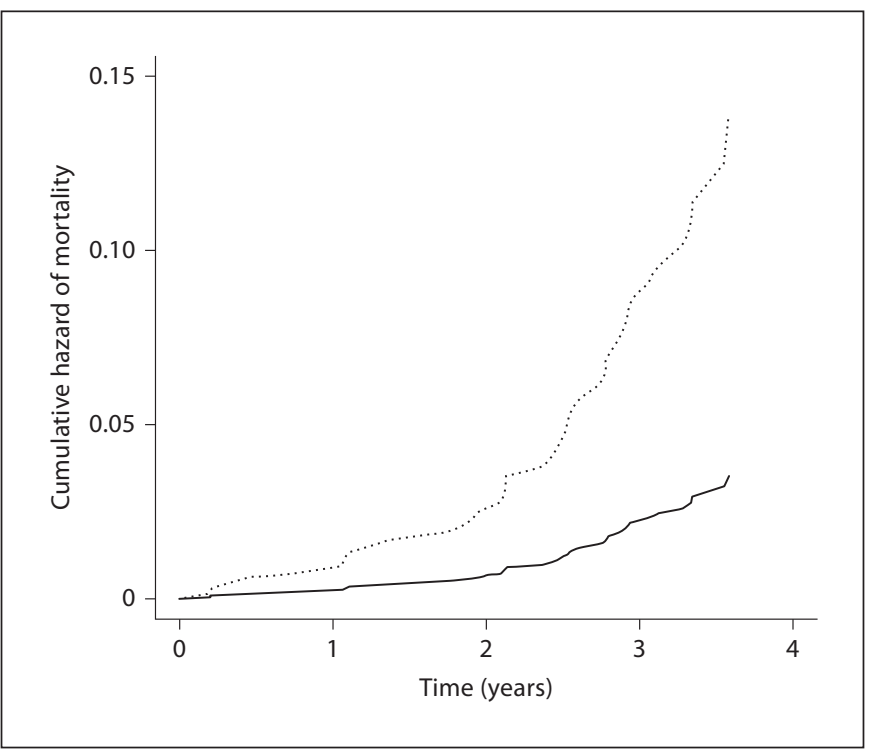

Fig. 1. Cumulative hazard of mortality for participants with poor (dotted line) versus good (solid line) decision making derived from a model adjusted for age, sex and education.

measured using 19 cognitive tests. The association of decision making with mortality persisted and was essentially unchanged in this analysis $(\mathrm{HR}=1.17,95 \% \mathrm{CI}=$ $1.04-1.31$, table 1), suggesting that it is relatively independent of cognition.

\section{Discussion}

We examined the association of decision making with mortality in more than 600 community-dwelling older persons without dementia. During up to 4 years of followup, poorer decision making was associated with an increased risk of death; more specifically, a person who performed poorly on the measure of decision making was about 4 times more likely to die over the study period compared to a person who performed well. Further, the association of decision making with mortality persisted after adjustment for the level of cognitive function. The finding that decision making is related to mortality in nondemented older persons after accounting for the level of cognitive function suggests that decision making is a complex behavior that warrants additional research focus as a potentially early and important harbinger of adverse health outcomes in old age.
Decision making has long been considered essential to maintenance of independence, health and well-being. In cross-sectional studies of older persons, decision making has been associated with several indicators of psychological health and well-being, including cognition, numeracy, social functioning, mood and indices of health $[2,3]$. Although it stands to reason that poor decision making would predict adverse health consequences, we are not aware of any longitudinal study that has examined whether poor decision making is associated with an increased risk of mortality in old age. This reflects an important gap in knowledge, as aging is a time when many important and impactful decisions are made just as cognitive function deteriorates and death approaches. It is widely accepted that older persons with dementia have impaired decision making, and emerging evidence suggests that persons with mild cognitive impairment, the preclinical phase of dementia, also exhibit poor decision making $[2,3,7,9,10,16]$. Further, compelling evidence suggests that even some 'cognitively healthy' persons exhibit impaired decision making [17]. In particular, the selective susceptibility of older persons to fraud raises questions about the quality of decision making in advanced age, and there are many other examples of older persons making poor healthcare (e.g. failure to enroll in necessary health benefit plans) and financial decisions (e.g. taking social security distributions early, poor retirement planning) $[4,18]$. The present results are the first that we are aware of that provide evidence that decision making may be an important determinant of health outcomes in old age.

It is noteworthy that there remains some debate regarding the extent to which decision making reflects an indicator of or proxy for cognition rather than a distinct construct. Notably, we excluded persons with overt dementia from our analyses. Further, we controlled for level of cognition using a detailed battery of 19 cognitive tests, and the association of decision making with mortality persisted. Thus, the effect of decision making on risk of death was above and beyond the level of cognition, which also predicts risk of death. These findings provide support for the validity of decision making as a distinct construct, a perspective that is supported by a large literature, mostly from behavioral economics and psychology, that shows that good decision making requires domain-specific knowledge, as well as personality traits such as patience and self-control $[2,3]$. Taken together, these studies suggest that simply having knowledge or computing power does not always result in better decisions. Given that decision making is a complex 
function of diverse characteristics, it may represent a behavior that is sensitive to even very early changes in cognition and may be a harbinger of adverse outcomes. Additional research is needed to better understand the correlates of decision making in old age and to determine whether decision making is related to other important health outcomes such as disability and dementia.

The basis of the association of decision making with mortality is unknown. One possibility is that this association is the result of comorbidities possibly due to a history of poor decision making. In other words, older persons who made poor decisions may have been sicker and thus at greater risk of death. Another possibility is that common, age-related neuropathologies (i.e. in Alzheimer disease and cerebrovascular disease), which are nearly ubiquitous in the brains of older persons, contribute to impairments in complex thinking and behavioral processes that underlie decision making $[19,20]$. Future studies are needed to elucidate the neurobiologic basis of the association of decision making with mortality, but we suspect that impaired decision making in older persons is not simply due to aging or cognitive impairment. Rather, it is the result of disease processes that either result from or contribute to poor decision making.

This study has several strengths, including the assessment of decision making in a large group of community-dwelling older persons who underwent a uniform clinical evaluation and in whom widely accepted criteria were used to exclude persons with dementia. In addition, we examined potential confounders of the association of decision making with mortality, including the level of global cognitive function measured via a well-established and detailed battery of tests. Limitations include the selected nature of the cohort, which may have restricted our range of scores on the decision making measure and may limit the generalizability of findings. In addition, the duration of follow-up was relatively short, although the results were significant and robust. Finally, although performance on an expanded decision making test that incorporated the items used here has been shown to be related to behaviors and abilities known to be associated with decision making (e.g. numeracy, health behaviors, motivation), further research is needed to determine the external validity of the decision making test used in this study $[2,3]$. That decision making was found to be associated with mortality even after controlling for cognition suggests that the measure used herein warrants further investigation. Future studies are needed to better understand the trajectory of decision making in aging and to examine the association of decision making with additional health outcomes.

\section{Acknowledgement}

This research was supported by National Institute on Aging grants R21AG30765 (to D.A. Bennett), R01AG17917 (to D.A. Bennett), R01AG34374 (to P.A. Boyle), R01AG33678 (to P.A. Boyle), and the Illinois Department of Public Health.

\section{Disclosure Statement}

The funding agencies had no role in the design, conduct, or interpretation of the data or preparation of the paper.

\section{References}

1 Denburg NL, Weller JA, Yamada TH, et al: Poor decision making among older adults is related to elevated levels of neuroticism. Ann Behav Med 2009;37:164-172.

- 2 Finucane ML, Gullion CM: Developing a tool for measuring the decision-making competence of older adults. Psychol Aging 2010;25:271-288.

3 Finucane ML, Mertz CK, Slovic P, Schmidt ES: Task complexity and older adults' decision-making competence. Psychol Aging 2005;20:71-84.
4 Agarwal S, Driscoll JC, Gabaix X, Laibson D: The age of reason: financial decisions over the life cycle and implications for regulation. Brookings Papers on Economic Activity 2009;2:51-117.

$\checkmark 5$ Grober E, Hall CB, Lipton RB, Zonderman $A B$, Resnick SM, Kawas C: Memory impairment, executive dysfunction, and intellectual decline in preclinical Alzheimer's disease. J Int Neuropsychol Soc 2008; 14:266-278.

6 Lai JM, Gill TM, Cooney LM, Bradley EH, Hawkins KA, Karlawish JH: Everyday decision-making ability in older persons with cognitive impairment. Am J Geriatr Psychiatry 2008;16:693-696.
7 Griffith HR, Belue K, Sicola A, et al: Impaired financial abilities in mild cognitive impairment: a direct assessment approach. Neurology 2003;60:449-457.

8 Karlawish J. Measuring decision-making capacity in cognitively impaired individuals. Neurosignals 2008;16:91-98.

-9 Marson DC, Sawrie SM, Snyder S, et al: Assessing financial capacity in patients with Alzheimer disease: a conceptual model and prototype instrument. Arch Neurol 2000;57: 877-884. 
10 Triebel KL, Martin R, Griffith HR, et al: Declining financial capacity in mild cognitive impairment: a 1-year longitudinal study. Neurology 2009;73:928-934.

- 11 Slovic P, Peters E, Finucane ML, Macgregor DG: Affect, risk, and decision making. Health Psychol 2005;24:S35-40.

12 Bennett DA, Schneider JA, Buchman AS Mendes de Leon C, Bienias JL, Wilson RS: The Rush Memory and Aging Project: study design and baseline characteristics of the study cohort. Neuroepidemiology 2005;25: 163-175.

13 Boyle PA, Wilson RS, Aggarwal NT, Tang Y, Bennett DA: Mild cognitive impairment: risk of Alzheimer disease and rate of cognitive decline. Neurology 2006;67:441-445.
14 Wilson RS, Barnes LL, Krueger KR, Hoganson G, Bienias JL, Bennett DA: Early and late life cognitive activity and cognitive systems in old age. J Int Neuropsychol Soc 2005; 11 : 400-407.

15 SAS 9.2 Help and Documentation (computer program). Cary, SAS Institute Inc, 2009.

16 Okonkwo OC, Wadley VG, Griffith HR, et al: Awareness of deficits in financial abilities in patients with mild cognitive impairment: going beyond self-informant discrepancy. Am J Geriatr Psychiatry 2008;16:650-659.
7 Denburg NL, Tranel D, Bechara A: The ability to decide advantageously declines prematurely in some normal older persons. Neuropsychologia 2005;43:1099-1106.

18 American Association of Retired Persons: Telemarketing Fraud and Older Persons: An AARP Survey. Washington, AARP, 1996.

19 Barnes LL, Schneider JA, Boyle PA, Bienias JL, Bennett DA: Memory complaints are related to Alzheimer disease pathology in older persons. Neurology 2006;67:1581-1585.

20 Bennett DA, Schneider JA, Arvanitakis Z, et al: Neuropathology of older persons without cognitive impairment from two communitybased studies. Neurology 2006;66:18371844. 